\title{
Urogynecology digest
}

\author{
Presented by Gans Thiagamoorthy
}

\section{What is the incidence of repeat pelvic floor surgery in women who have undergone childbirth after previous pelvic floor surgery? \\ Pradhan A, Tincello D, Kearney $R$ (2013) Childbirth after pelvic floor surgery: analysis of Hospital Episode Statistics in England, 2002-2008. BJOG 120:200-204}

Pregnancy and childbirth are significant factors in the aetiology of pelvic floor dysfunction (PFD). The authors attempted to assess the effect of childbirth on previous surgery for PFD using repeat surgery for PFD as the primary outcome measure. Data were collected via 'Hospital Episode Statistics', an online database of all episodes of admission to hospitals for National Health Service (NHS) funded care in the UK. The database was searched for women between the ages of 20 and 44 years who had undergone surgery for PFD (pelvic organ prolapse or urinary incontinence) and then a subsequent delivery between April 2002 and April 2008. A total of 603 women fit the criteria with 671 delivery episodes. In this group, 42 women had 50 subsequent episodes of pelvic floor surgery before April 2008. The incidence of repeat surgery episodes for PFD was calculated as higher after a vaginal delivery (30 of 221, $13.6 \%$ ) than after caesarean (20 of 450, 4.4\%) with an odds ratio of 3.38 [95\% confidence interval (CI) 1.87-6.10].

The authors have sought to collect data to assist in informative discussion with women regarding future fertility aspirations, mode of delivery and associated risks. This is a retrospective review over a relatively short time scale without a control group which limits extrapolating these data to the general population. The indications for the primary and subsequent pelvic floor surgery have been assumed to be recurrence rather than new occurrence of PFD. To illustrate an example, posterior repair after previous mid-urethral sling does not represent failure of the sling procedure. Therefore one needs to be cautious in interpreting the concluding message of the authors that these data "may indicate a protective effect of abdominal delivery." Nonetheless, this paper adds some further evidence to the existing hypothesis that caesarean sections may reduce deleterious effects of childbirth on previous surgery for PFD.

\section{Could interstitial cystitis/bladder pain syndrome lead to bladder cancer? \\ Keller J, Chiou HY, Lin HC (2013) Increased risk of bladder cancer following diagnosis with bladder pain syndrome/interstitial cystitis. Neurourol Urodyn 32:58-62}

Data were also collected via a national database, this time from Taiwan's National Health Insurance (NHI) database which records all inpatient and outpatient medical benefit claims of more than $98 \%$ of Taiwan's population. Women with a new diagnosis of interstitial cystitis/bladder pain syndrome (IC/BPS) and who were started on intravesical hyaluronic acid (Cystistat ${ }^{\mathrm{TM}}$ ) between January 2006 and December 2007 were included. The authors explain that the NHI system only allows Cystistat ${ }^{\mathrm{TM}}$ to be prescribed for proven IC/BPS and requires the use of strict diagnostic criteria including potassium sensitivity test, hydrodistension test and cystoscopy, thus reducing the risk of incorrect diagnosis including pre-existing bladder cancer (BC). The study included 7,562 patients with IC/BPS. For every patient with IC/BPS, 3 control patients matched for geographical location and level of urbanisation were also assessed, 22,686 women. To assess whether having a urological disease increased the risk of diagnosis of $\mathrm{BC}$, a final group of 5,921 women with urinary incontinence (UI) was also studied. All patients were followed for 3 years.

Of 7,562 women with IC/BPS, 48 (0.63\%) developed BC compared to $48(0.21 \%)$ of 22,686 controls and 18 $(0.30 \%)$ of 5,921 women with UI. This equates to an incidence rate of $\mathrm{BC}$ per 1,000 person years of 2.12 (95\% CI 1.58-2.78), 0.70 (95\% CI 0.2-0.92) and 1.01 (95\% CI 0.62-1.57), respectively. The hazard ratio, used to present the risk of $\mathrm{BC}$ during the 3-year follow-up for the IC/BPS group compared to the control group, was 2.95 (95\% CI 1.97-4.41, $p<0.01)$.

The study benefits from a large sample size and stringent diagnostic criteria. IC/BPS and BC have very similar presentations and there is a chance of initial misdiagnosis. This was reduced by the NHI criteria for the prescription of Cystistat $^{\mathrm{TM}}$ requiring a prior cystoscopy, but it should be noted that cytology was optional and the authors do not mention any of the women undergoing biopsies at 
cystoscopy. The risk of surveillance bias was well controlled looking at the group with UI. The duration of follow-up was 3 years so may have under-represented the chance of developing $\mathrm{BC}$ since diagnosis of either IC/BPS or UI. The final point raised by the authors is that all patients in the IC/BPS arm were treated with Cystistat ${ }^{\mathrm{TM}}$ and their study would not differentiate the effects of the medication versus the IC/BPS disease process on the risk of developing BC. The significant increase in BC after diagnosis of IC/BPS is novel and must be investigated further, especially as the time interval was less than only 3 years. This will have drastic implications for the risk stratification of $\mathrm{BC}$ and also the counselling and management of IC/BPS if reaffirmed.

\section{How do you manage voiding dysfunction after MIS insertion?}

Agnew G, Dwyer PL, Rosamilia A, Edwards G, Lee JK (2012) Functional outcomes for surgical revision of synthetic slings performed for voiding dysfunction: a retrospective study. Eur J Obstet Gynecol Reprod Biol 163:113-116

There is a lack of clear guidance regarding the management of voiding dysfunction after insertion of a minimally invasive suburethral sling (MIS). The outcomes of different methods of sling revision to treat this complication are evaluated via a retrospective study. Women with bothersome symptoms of voiding dysfunction and a post-void residual (PVR) of $>150 \mathrm{ml}$ who underwent MIS revision between 2000 and 2010 at two university hospitals were included. Revision was performed by either dividing or partially excising the MIS lateral to the urethra and in some cases a concomitant continence procedure was also carried out. Cases where MIS was only loosened were excluded. Sixty-three women underwent MIS revision. Success, defined as PVR $<150 \mathrm{ml}$, was achieved in $87 \%$. Persistence of voiding dysfunction was similar where MIS was divided (10.9 \%) or partially excised $(7.7 \%)$ and highest with a concomitant continence procedure $(50 \%)$. Subsequent re-operation rates for stress urinary incontinence (SUI) were $2.2 \%$ for MIS division, $23.1 \%$ for partial excision and nil with the concomitant procedure. The increase in re-operation after MIS excision was significantly higher $(p=0.04)$. There was no significant difference in outcome depending on the time interval between initial insertion and subsequent MIS revision.

Agnew et al. describe a retrospective analysis of 63 women undergoing three different treatments for voiding dysfunction post MIS but only 4 women underwent the third procedure (concomitant continence surgery). Due to the low incidence of voiding dysfunction after MIS it is difficult to carry out a randomised controlled trial (RCT) to assess the best management plan for women with these symptoms. Although bothersome symptoms of voiding dysfunction were part of the criteria to identify the cohort, improvement in these symptoms was not part of the definition of success. Data on the total number of sling insertions in the units over the 10 years would have also been useful to frame the voiding dysfunction rate in their population. With the low rate of persistent voiding dysfunction (11\%) and significantly lower risk of recurrent SUI (2.2\%) simple division of MIS alone would seem the ideal recommendation. Of the 63 women who required MIS revision for voiding dysfunction, $49 \%$ had undergone concomitant pelvic organ prolapse (POP) surgery whilst the remainder had not. It is worth noting that this study also adds further weight to the growing evidence base that there is no difference in urinary function outcomes with concomitant MIS and POP surgery.

The findings of this study thus are very important, providing guidance for surgeons and information for the preoperative counselling of patients.

Gans Thiagamoorthy

King's College Hospital, London SE5 9RS, UK

e-mail: gans.t@nhs.net 